\title{
Long-term use of a once-a-day fentanyl citrate transdermal patch in lung cancer patients
}

\author{
OSAMU TAKAKUWA, TETSUYA OGURI, KEN MAENO, HIROKI MURASE, TAKAMITSU ASANO, \\ HIROYA ICHIKAWA, YUKO KAWAGUCHI, TAKEHIRO UEMURA, HIROTSUGU OHKUBO, \\ MASAYA TAKEMURA and AKIO NIIMI \\ Department of Respiratory Medicine, Allergy and Clinical Immunology, \\ Nagoya City University Graduate School of Medical Sciences, Nagoya, Aichi 467-8601, Japan
}

Received April 5, 2014; Accepted January 23, 2015

DOI: $10.3892 / \mathrm{ol} .2015 .3022$

\begin{abstract}
Transdermal fentanyl is widely administered as an analgesic therapy for cancer patients. Recently, a novel fentanyl citrate transdermal patch was developed in Japan, which is the first such patch that requires changing only once a day. The patch releases more stable serum fentanyl concentrations and results in less frequent adverse skin symptoms compared with the conventional 72-h transdermal fentanyl patch. A previous study has reported the short-term analgesic effects and safety of this transdermal patch. However, the long-term efficacy has yet to be determined. Therefore, the present study retrospectively investigated the adverse effects and outcomes of treatment with this product in 46 lung cancer patients. In total, 35 (76\%) patients were able to continue the treatment until the end of the observation period, including $32(69 \%)$ who succumbed to the disease and three (7\%) who were transferred from Nagoya City University Hospital to a hospice. Of the 11 (24\%) discontinued cases, six were changed to a course of injectable opioids for the palliation of terminal symptoms, including dyspnea. Adverse effects were observed in 11 (24\%) cases, but discontinuation due to adverse effects occurred in only four cases. Therefore, the present study indicates that palliative treatment with the once-a-day fentanyl citrate transdermal patch is well-tolerated by lung cancer patients.
\end{abstract}

\section{Introduction}

Pain is reported in a minimum of one-third of newly-diagnosed cancer patients and in $65-85 \%$ of patients experiencing advanced cancer $(1,2)$. Opioids are the primary agents used

Correspondence to: Dr Tetsuya Oguri, Department of Respiratory Medicine, Allergy and Clinical Immunology, Nagoya City University Graduate School of Medical Sciences, 1 Kawasumi, Mizuho-cho, Mizuho-ku, Nagoya, Aichi 467-8601, Japan

E-mail: t-oguri@med.nagoya-cu.ac.jp

Key words: fentanyl citrate, lung cancer, palliative treatment, transdermal patch, terminal care, transdermal fentanyl to treat moderate to severe cancer-associated pain, with oral morphine and oxycodone being administered as the predominant analgesics for cancer patients until the 1990s (3). However, these oral opioids frequently induce digestive side-effects, including nausea and constipation, and advanced-stage cancer patients often find oral intake difficult due to a number of side-effects, including cachexia and mechanical obstruction. Therefore, adhering to oral agent regimens may be challenging for advanced cancer patients (3).

As an alternative to oral administration, the transdermal route is a noninvasive method of delivering therapeutic agents. For example, transdermal fentanyl provides a similar analgesic efficacy to oral morphine, as well being associated with a low incidence of adverse gastrointestinal events (4) and greater patient satisfaction (5). Therefore, a number of guidelines currently recommend transdermal fentanyl for the treatment of cancer-associated pain, particularly for patients who are unable to swallow (6-8). A novel transdermal patch containing fentanyl citrate was recently developed in Japan, which is the first such patch to require changing only once a day. This novel once-a-day product produced more stable serum fentanyl concentrations and less frequent occurrences of adverse skin symptoms compared with the conventional 72-h transdermal fentanyl patch (9). Furthermore, a previous study reported the short-term analgesic and adverse effects of this once-a-day fentanyl citrate transdermal patch in lung cancer patients when the product was initially introduced. Decreased pain scores were observed subsequent to switching from oral analgesics in $\sim 60 \%$ of patients and the adverse effects appeared to be mild (10). However, the follow-up period of the aforementioned study was short. Therefore, the longer-term use of this once-a-day fentanyl citrate transdermal patch requires evaluation. In the present study, the long-term use of a once-a-day fentanyl citrate transdermal patch was retrospectively examined in lung cancer patients.

\section{Patients and methods}

Between July 2011 and December 2013, 46 lung cancer patients that had undergone treatment with the once-a-day fentanyl citrate transdermal patch at Nagoya City University Hospital were enrolled in the present study. The patients 
included 38 males and 8 females, with ages ranging between 37 and 83 years (median age, 68.2 years). The most common pathological type was adenocarcinoma (48\%). All patients had a clinical cancer stage of IV with the exception of one patient with a stage of IIIb (Table I). All patients commenced the fentanyl citrate patch treatment during hospitalization and bone metastasis was the most frequent cause of pain. The most common reason for switching between an oral opioid and a once-a-day fentanyl citrate transdermal patch was difficulty with oral intake, followed an adverse effect caused by the oral opioid. The oral opioid was typically oral oxycodone and, in more than one-half of the patients, the switching dose of the once-a-day fentanyl citrate transdermal patch was $1 \mathrm{mg}$. In all patients, rescue dose of opioid was prepared and NSAIDs was used as combination drug therapy in 21 patients.

Commercialized optimal dose conversion values were used to determine the fentanyl citrate patch dosage required from the oral opioid dose administered (Table II). The adverse effects of opioid therapy were evaluated by healthcare workers, according to the National Cancer Institute Common Toxicity Criteria version 4.0 (11). Treatment after the initiation of a fentanyl citrate transdermal patch was managed by each primary physician and the treatment outcome, including the treatment length, any reasons for changes to treatment and followed treatment and post-fentanyl citrate transdermal patch treatment, was obtained from each clinical record. The treatment length for the fentanyl citrate transdermal patch was between 1 and 516 days (mean, 53.5 days). The present study was approved by the ethics committee of Nagoya City University Graduate School of Medical Sciences (Nagoya, Aichi, Japan) and written informed consent was obtained from all patients.

\section{Results}

Adverse effects of the once-a-day fentanyl citrate transdermal patch (Table III). Adverse effects of the once-a-day fentanyl citrate transdermal patch were observed in 11 out of $46(24 \%)$ patients. The most common adverse events were nausea and drowsiness, observed in three patients (7\%) each, and all adverse events exhibited were within grades I and II, excluding one case of grade III nausea. There was no incidence of a rash that required medication. Furthermore, an adverse hepatic effect was observed in one patient, in which a grade II increase in aspartate transaminase (AST) and alanine transaminase (ALT) levels occurred nine days after commencing treatment with the transdermal patch. The fentanyl citrate transdermal patch treatment was subsequently terminated and oxycodone hydrochloride hydrate treatment commenced, causing the hepatic disorder to resolve and the AST and ALT levels to return to within the normal ranges (AST, normal range, 13-33 U/1; ALT, normal range, 6-30 U/1).

Treatment outcome with a once-a-day fentanyl citrate transdermal patch (Table IV). Use of the once-a-day fentanyl citrate transdermal patch was continued until the end of the observation period in 35 out of $46(76 \%)$ patients, including 32 who succumbed to the disease $(69 \%)$ and three who were transferred from Nagoya City University Hospital to a hospice (7\%). Furthermore, the mean survival period, from commencing the
Table I. Patient characteristics ${ }^{\mathrm{a}}$.

\begin{tabular}{lr}
\hline Characteristic & $\mathrm{n}(\%)$ \\
\hline Gender & \\
Male & $38(83)$ \\
Female & $8(17)$ \\
Pathological type & \\
Adenocarcinoma & $22(48)$ \\
Squamous cell carcinoma & $9(20)$ \\
Small cell & $6(13)$ \\
Other types & $9(20)$ \\
Location of pain & \\
Bone metastasis & $24(52)$ \\
Local progression & $14(30)$ \\
Other & $8(17)$ \\
Reason for switching to FP & \\
Difficulty in oral intake & $29(63)$ \\
AE of opioid & $16(35)$ \\
Other & $1(2)$ \\
Previous opioid treatment & \\
Oxycodone & $41(89)$ \\
Morphine & $5(11)$ \\
Initial dose of FP, mg & \\
1 & \\
2 & \\
4 & \\
\hline
\end{tabular}

${ }^{a}$ Median patient age, 68.2 years (range, 37-83 years). FP, fentanyl citrate transdermal patch; AE, adverse effect.

Table II. Conversion between the dose for oral opioids and the dose for the once-a-day type fentanyl citrate patch.

\begin{tabular}{lcc}
\hline $\begin{array}{l}\text { Oral } \\
\text { morphine, mg }\end{array}$ & $\begin{array}{c}\text { Oral } \\
\text { oxycodone, mg }\end{array}$ & $\begin{array}{c}\text { Fentanyl citrate } \\
\text { patch, mg }\end{array}$ \\
\hline$\leq 19$ & $\leq 29$ & 1 \\
$20-59$ & $30-89$ & 2 \\
$60-99$ & $90-149$ & 4 \\
$100-139$ & $150-209$ & 6 \\
\hline
\end{tabular}

Table III. Most frequent adverse events during treatment.

\begin{tabular}{lcc}
\hline Event & $\mathrm{n}(\%)$ & Grade $\geq 3, \mathrm{n}(\%)$ \\
\hline Nausea & $3(7)$ & $1(2)$ \\
Somnolence & $3(7)$ & $0(0)$ \\
Constipation & $2(4)$ & $0(0)$ \\
Delirium & $1(2)$ & $0(0)$ \\
Anorexia & $1(2)$ & $0(0)$ \\
ALT/AST level increased & $1(2)$ & $0(0)$ \\
\hline
\end{tabular}

ALT, alanine transaminase; AST, aspartate transaminase. 
Table IV. Outcome of FP treatment.

\begin{tabular}{|c|c|c|c|}
\hline Outcome of FP treatment & $\mathrm{n}(\%)$ & Length of FP treatment ${ }^{\mathrm{a}}$, days & Survival, days \\
\hline \multicolumn{4}{|l|}{ Continuation until end of observation } \\
\hline Due to mortality & $32(69)$ & $62.7 \pm 113.9$ & $62.7 \pm 113.9$ \\
\hline Due to transfer from hospital to hospice & $3(7)$ & $29.7 \pm 21.0$ & ND \\
\hline Total & $35(76)$ & $59.8 \pm 109.2$ & ND \\
\hline \multicolumn{4}{|l|}{ Change in treatment } \\
\hline For palliation of terminal-stage symptoms & $6(13)$ & $49.7 \pm 57.1$ & $56.8 \pm 62.6$ \\
\hline Due to adverse effects of FP & $4(9)$ & $12.5 \pm 6.4$ & $32.0 \pm 9.9$ \\
\hline For control of cancer pain & $0(0)$ & ND & ND \\
\hline Other & $1(2)$ & $72.0 \pm 0.0$ & $112.0 \pm 0.0$ \\
\hline Total & $11(24)$ & $41.3 \pm 48.8$ & $54.2 \pm 54.8$ \\
\hline
\end{tabular}

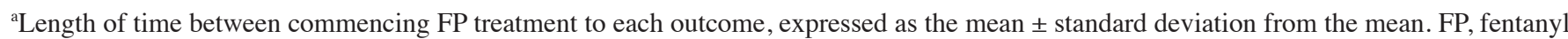
citrate transdermal patch; ND, not determined.

once-a-day fentanyl citrate transdermal patch to mortality, was 62.7 days.

In the present study, 39 out of $46(85 \%)$ patients required an increase in the dose of the fentanyl citrate transdermal patch; however, no patients required the treatment to be terminated due to a lack of pain control. Of the 11 patients that discontinued treatment, six $(13 \%)$ changed treatment strategy to injectable opioids for the palliation of terminal symptoms, such as dyspnea. Of these six cases, five underwent morphine hydrochloride injections following treatment with the fentanyl citrate transdermal patch. In addition, the mean survival period subsequent to changing between the once-a-day fentanyl citrate transdermal patch and injectable opioids was $\sim 7$ days, which was calculated by subtracting the mean survival during fentanyl treatment (49.7 days) from the mean total survival (56.8 days).

Furthermore, four patients $(9 \%)$ did not continue with fentanyl treatment due to adverse effects, including two patients developing nausea, one developing somnolence and one developing an increased AST/ALT ratio, and one patient demonstrated difficulty with oral rescue opioid intake due to mechanical obstruction of the esophagus.

\section{Discussion}

The use of oral opioids is recommended as a basic strategy for the treatment of cancer-associated pain, according to the World Health Organization guidelines (3). However, advanced cancer patients commonly find oral intake challenging due to a number of side-effects, including cachexia or mechanical obstruction, thus, adhering to oral therapy regimens until mortality is often challenging (12). In the present study, 32 out of $46(69 \%)$ patients continued treatment with the once-a-day fentanyl citrate transdermal patch to mortality. In the cases that required opioid injections subsequent to treatment with the fentanyl citrate transdermal patch, the mean survival time from commencing injectable opioid therapy was just one week. These results indicate that treatment with a once-a-day fentanyl citrate transdermal patch may be continued for the majority of the time until mortality. Furthermore, a previous study reported the safety and analgesic effects of this treatment when switching from oral opioid treatment (10). The present and previous results indicate that the once-a-day fentanyl citrate transdermal patch may be useful for the treatment of lung cancer patients experiencing pain.

Nausea, vomiting and drowsiness have previously been reported as adverse events in $>5 \%$ of patients treated with the once-a-day fentanyl citrate transdermal patch (9), similar to the proportion affected by traditional transdermal fentanyl therapy. Consistent with this previous report, the present study observed nausea and drowsiness as the most common adverse effects (9). However, the majority of patients in the present study experienced mild adverse effects. As numerous patients in the current study were in the late stages of lung cancer, the degree and frequency of the adverse effects were considered tolerable. However, one patient developed an increase in the ALT/AST level. Lung cancer often results in liver metastases (13), and numerous agents are concomitantly used in the treatment of such patients, therefore, when liver damage is identified, it is necessary to consider the fentanyl patch as a potential cause.

Termination of the fentanyl citrate transdermal patch was required in six cases $(13 \%)$ in favor of injectable opioids for the palliation of symptoms, including dyspnea. The efficacy of morphine for the palliation of dyspnea in cancer patients is well-documented $(14,15)$, and a recent randomized crossover clinical trial identified that the administration of oral transmucosal fentanyl citrate does not appear to improve exertional dyspnea in patients with advanced cancer, compared with the placebo (16). In lung cancer, the majority of patients exhibit dyspnea in the terminal stage (17). Therefore, the use of morphine may continue to be considered when dyspnea occurs during treatment with the fentanyl citrate transdermal patch.

Limitations of the present study include its retrospective nature and the inclusion of just one clinical study center. Furthermore, the types of underlying disease were limited to lung cancer and the patient cohort was limited to hospital inpatients. Despite the once-a-day fentanyl citrate patch being utilized by homecare patients and outpatients, these 
patients were not included in the current study. Furthermore, the present study included three patients who could not be followed up until mortality as they changed the hospital at which they were undergoing treatment. Therefore, it is possible that other adverse effects occurred during their remaining survival time.

The results of the present study indicate that the once-a-day fentanyl citrate transdermal patch is an acceptable long-term strategy for the treatment of cancer-associated pain control in lung cancer patients. Additional prospective clinical trials are required to evaluate the clinical efficacy of the once-a-day fentanyl citrate patch.

\section{References}

1. Cleeland CS, Gonin R, Hatfield AK, et al: Pain and its treatment in outpatients with metastatic cancer. N Engl J Med 330: 592-596, 1994.

2. van den Beuken-van Everdingen MH, de Rijke JM, Kessels AG, Schouten HC, van Kleef $M$ and Patijn J: Prevalence of pain in patients with cancer: a systematic review of the past 40 years. Ann Oncol 18: 1437-1449, 2007.

3. World Health Organization: Cancer pain relief: with a guide to opiod availability. 2nd edition. World Health Organization, Geneva, Switzerland, pp12-38, 1996

4. Mercadante S, Porzio G, Ferrera P, et al: Sustained-release oral morphine versus transdermal fentanyl and oral methadone in cancer pain management. Eur J Pain 12: 1040-1046, 2008.

5. Payne R, Mathias SD, Pasta DJ, Wanke LA, Williams R and Mahmoud R: Quality of life and cancer pain: satisfaction and side effects with transdermal fentanyl versus oral morphine. J Clin Oncol 4: 1588-1593, 1998

6. Caraceni A, Hanks G, Kaasa S, et al; European Palliative Care Research Collaborative (EPCRC); European Association for Palliative Care (EAPC): Use of opioid analgesics in the treatment of cancer pain: evidence-based recommendations from the EAPC. Lancet Oncol 13: e58-e68, 2012.

7. National Collaborating Centre for Cancer (UK): Opioids in Palliative Care: Safe and Effective Prescribing of Strong Opioids for Pain in Palliative Care of Adults. Cardiff (UK): National Collaborating Centre for Cancer (UK); National Institute for Health and Clinical Excellence: Guidance, May 2012
8. Hanks GW, Conno F, Cherny N, et al; Expert Working Group of the Research Network of the European Association for Palliative Care: Morphine and alternative opioids in cancer pain: the EAPC recommendations. Br J Cancer 84: 587-593, 2001.

9. Miyazaki T, Namiki A, Ogawa S, et al: A phase II clinical study of once-a-day fentanyl citrate patch in patients with cancer pain - switching from once-every-three-days fentanyl patch to once-a-day fentanyl citrate patch. Gan To Kagaku Ryoho 37: 1747-1752, 2010 (In Japanese).

10. Takakuwa O, Oguri T, Maeno K, et al: Analgesic effect of switching from oral opioids to a once-a-day fentanyl citrate transdermal patch in patients with lung cancer. Am J Hosp Palliat Care 30: 726-729, 2013.

11. National Cancer Institute Common Toxicity Criteria version 4.0. http://evs.nci.nih.gov/ftp1/CTCAE/About.html. Accessed April 17, 2012.

12. Shimizu Y, Nagaya N, Isobe $T$, et al: Increased plasma ghrelin level in lung cancer cachexia. Clin Cancer Res 9: 774-778, 2003.

13. Vigano A, Donaldson N, Higginson IJ, et al: Quality of life and survival prediction in terminal cancer patients: a multicenter study. Cancer 101: 1090-1098, 2004.

14. Bruera E, MacEachern T, Ripamonti C and Hanson J: Subcutaneous morphine for dyspnea in cancer patients. Ann Intern Med 119: 906-907, 1993.

15. Mazzocato C, Buclin T and Rapin $\mathrm{CH}$ : The effects of morphine on dyspnea and ventilatory function in elderly patients with advanced cancer: a randomized double-blind controlled trial. Ann Oncol 10: 1511-1514, 1999.

16. Pinna MA, Bruera E, Moralo MJ, Correas MA and Vargas RM: A randomized crossover clinical trial to evaluate the efficacy of oral transmucosal fentanyl citrate in the treatment of dyspnea on exertion in patients with advanced cancer. Am J Hosp Palliat Care: Nov 20, 2013 (Epub ahead of print).

17. Claessens MT, Lynn J, Zhong Z, et al: Dying with lung cancer or chronic obstructive pulmonary disease: insights from SUPPORT. Study to understand prognoses and preferences for outcomes and risks of treatments. J Am Geriatr Soc 48 (Suppl): S146-S153, 2000 . 